\title{
Status of MR in South Africa
}

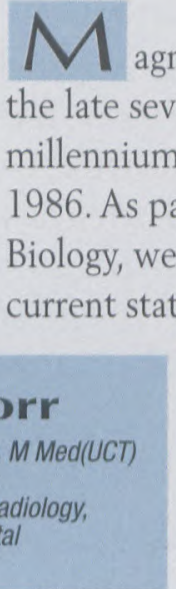

agnetic resonance imaging (MRI) has developed from an experimental imaging modality in the late seventies to an essential imaging investigation in radiology at the beginning of the millennium. The first MR scanner was installed by the Medical Research Council in Cape Town in 1986. As part of the Winter School of the South African Association of Physicists in Medicine and Biology, we sent out questionnaires to the main MR vendors in South Africa to determine the current status of MR in this country. In the questionnaire we asked questions about the number,

\section{Peter Corr} $M B C h B$, FFRad(D)SA, FRCR, M Med(UCT)

Head: Department of Radiology, University of Natal

type, field strength of scanners and their geographic distribution. We recorded the number of patients scanned per week, the breakdown per anatomical region, and the manpower of radiologists, radiographers and physicists involved in MR.

We received replies from all five MR vendors. In June 2000 there were 56 MR scanners, although this is likely to be increased to 60 by the end of 2000. Fifty-four are in the private sector $(96 \%)$ and two are in the public sector (Universitas and Wentworth Hospitals). The number of MR scanners in public hospitals is likely to be increased by two to a total of four by year-end. Most scanners are relatively new, with an average age of around three years. The oldest, which is now 10 years old, is being replaced. There have been two trends in the MR market in recent years: a trend towards open systems and a recent trend towards high field, high gradient MR systems. There are $171.5 \mathrm{~T}$ strength scanners (30\%), many with strong gradients with fast slew rates. There are 11 open $0.2 \mathrm{~T}$ systems $(20 \%)$, many in smaller cities and towns. Currently 24 scanners (43\%) are in Gauteng, nine in the Cape Town area and four in Durban. The remaining 29 $(52 \%)$ are evenly distributed in the smaller cities and towns throughout the country.

The patient workload varied from 15 to 60 patients per five-day week for each scanner. Anatomical regions scanned in an average week were: spine $50 \%$, brain $20 \%$, musculoskeletal $20 \%$, cardiac and peripheral vessels $10 \%$. It was difficult to assess how many radiologists and radiographers were actively performing MR imaging as this information was not forthcoming. There are about 10-12 medical physicists interested in and involved with MR.

There are a number of encouraging features from this small survey. South Africa is well placed internationally with almost 60 scanners working, many of which are relatively new technology. Many practices have formed alliances with public/academic departments to provide public patient imaging services and training of registrars and radiographers. The installed base is relatively new and many of the high field systems are capable of performing advanced cardiac and neurovascular sequences like cerebral diffusion and perfusion imaging for stroke investigations.

The future growth in MR will be in cardiac and peripheral vascular applications. Coronary MR imaging is becoming a reality and the role of cardiology in MR imaging will have to be addressed by the radiology society and speciality. Will MR go the same way as echocardiography? For radiologists to protect their referral base, they will have to keep up-to-date with the recent advances in MR technology and ultra fast imaging. This is no easy task considering the rapid advance of technology with new developments in hardware and software sequences arriving on the market almost monthly.

\section{Peter Corr}

\section{Editor}

